\title{
Treatment of Superior Vena Cava (SVC) Syndrome and Inferior Vena Cava (IVC) Thrombosis in a Patient with Colorectal Cancer: Combination of SVC Stenting and IVC Filter Placement to Palliate Symptoms and Pave the Way for Port Implantation
}

\author{
Alexander Sauter $\cdot$ Jürgen Triller $\cdot$ Felix Schmidt . \\ Ralph Kickuth
}

Published online: 29 June 2007

(C) Springer Science+Business Media, LLC 2007

\begin{abstract}
Thrombosis of the inferior vena cava is a lifethreatening complication in cancer patients leading to pulmonary embolism. These patients can also be affected by superior vena cava syndrome causing dyspnea followed by trunk or extremity swelling. We report the case of a 61year-old female suffering from an extended colorectal tumor who became affected by both of the mentioned complications. Due to thrombus formation within the right vena jugularis interna, thrombosis of the inferior vena cava, and superior vena cava syndrome, a combined interventional procedure via a left jugular access with stenting of the superior vena cava and filter placement into the inferior vena cava was performed As a consequence, relief of the patient's symptoms, prevention of pulmonary embolism, and paving of the way for further venous chemotherapy were achieved.
\end{abstract}

Keywords Interventional procedures - Inferior vena cava filter $\cdot$ Pulmonary embolism $\cdot$ Superior vena cava syndrome - Vena cava stent $\cdot$ Venous thrombosis

\section{Introduction}

In industrial nations like the United States, colorectal cancer is the second leading cause of death from cancer [1]. Such a systemic disease may lead to a 4.1 -fold elevated risk of a venous thrombosis [2]. The chance of a venous thromboembolism (VTE) is specifically high in patients

A. Sauter · J. Triller $\cdot$ F. Schmidt $\cdot$ R. Kickuth $(\bowtie)$ Department of Diagnostic, Interventional and Pediatric Radiology, Inselspital, University of Berne, Freiburgstrasse 20, CH-3010 Berne, Switzerland

e-mail: ralph.kickuth@insel.ch treated with a combination of fluorouracil and leucovorin calcium because of colorectal cancer ( $15 \%$ of patients were affected) [3]. The 1-year survival rate of cancer patients afflicted by such an event is $12 \%$, versus $36 \%$ in patients without embolism [4]. Pathogenetic mechanisms of hypercoagulability are the production of procoagulants and the activation of endogenous prothrombotic factors by the tumor itself $[5,6]$.

Besides liver and lung metastasis, colorectal cancer can also affect the adrenal gland, which constitutes only $13.5 \%$ of all adrenal metastasis of primary tumors [7]. More common in renal cell carcinoma or adrenocortical carcinoma, an adrenal metastasis of a colorectal cancer can also invade the inferior vena cava (IVC), causing a dangerous situation requiring intervention, like a vena cava filter, to prevent pulmonary embolism $[8,9]$.

Another rare complication of colorectal cancer is superior vena cava syndrome (SVCS), causing obstruction of the superior vena cava (SVC) [10]. Owing to its thin wall and the low intravascular pressure, the SVC has only a low resistance to an extrinsic compression, and thrombus formation is easily possible. This may result in congestion and edema of the face and upper thorax, as well as symptoms like dyspnea, dysphagia, cognitive dysfunction, and headache. Therapeutical options for patients with SVCS having already received chemotherapy and radiation therapy are limited. Venous stenting is usually helpful in such palliative situations, where life quality is paramount, due to the simplicity of the procedure and rapid relief of symptoms [11].

This rare case therefore highlights the subsequent management of SVCS and IVC thrombosis with stent placement and filter insertion in a patient with metastatic rectal cancer to relieve symptoms, prevent pulmonary embolism, and pave the way for port implantation. 


\section{Case Report}

Our institutional review board does not require approval for publication of retrospective case reports. A 61-year-old female with a history of metastatic rectal cancer was referred to our hospital for surgical port implantation. In the distant past, primary staging had yielded malignant pulmonary and mediastinal spread, and the patient preoperatively underwent two cycles of chemotherapy combined with external-beam radiotheratpy ( $45 \mathrm{~Gy}$ ) to the rectum. Four months after primary diagnosis, a complete mesorectal excision and low anterior resection with colorectal anastomosis was carried out by our visceral surgeons. The pathological analysis revealed a pT3, pN1, M1, G2 stadium of the tumor. Three months after surgery, suspicion of metastases of the liver was confirmed by magnetic resonance imaging of the abdomen showing two lesions in segments V/VI with a diameter of 5.4 $\mathrm{cm}$ and VII/VIII with diameter of $1.7 \mathrm{~cm}$. As a consequence, hemihepatectomy of the right lobe was scheduled 1 day later.

The situation seemed to be stable, when a PET-CT scan exposed a progression of the pulmonary and mediastinal lesions 21 months later. These findings led to an expansive resection of the right lung by our thoracic surgeons (upper lobe resection, two-times atypical resection of the middle lobe, two-times atypical resection of the lower lobe, mediastinal retrocaval lymphadenectomy), with an R0 situation of all metastases, except for the retrocaval manifestation, with an R1 situation. One day postoperatively, CT showed a thrombosis of the right internal jugular vein and the right brachiocephalic vein. Treatment of venous thrombosis was initiated with oral anticoagulation therapy. Over the next 3 months, increasing symptoms of SVCS with swollen head and arm veins, headache, dyspnea, and livid face occurred. Another CT examination was performed that further exhibited thrombosis of the right internal jugular vein and the right brachiocephalic vein, but also recurrent mediastinal tumor spread and new onset of an inhomogeneous hypodense metastatic lesion of the right adrenal gland with an extent of $7.0 \times 7.2 \mathrm{~cm}$. As a consequence, the Department of Oncology was consulted and recommended palliative chemotherapy according to the FOLFOX scheme. On the grounds of the oral anticoagulation and thrombosis, no port could be installed, and the first cycle of chemotherapy had to be arranged via a peripheral access.

One month later, due to persisting symptoms and the fact that an efficient port would be a major part of the continuing therapy, the patient was referred to our interventional radiology department for SVC stent implantation. The major intention of this approach was to relieve symptoms and contemporaneously pave the way for port implantation. At this point in time oral anticoagulation had been stopped. We initiated a follow-up CT to evaluate the actual situation before stent implantation. The scan showed that the metastasis

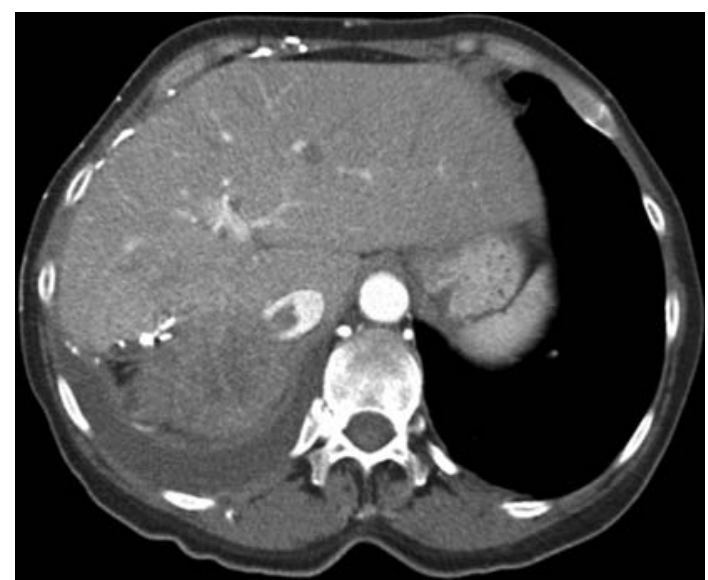

Fig. 1 Contrast-enhanced CT scan shows metastasis of the adrenal gland with tumor invasion and partial thrombosis of the IVC

of the adrenal gland now reached $7.2 \times 8.4 \mathrm{~cm}$, with tumor invasion into the right suprarenal vein and renal vein, and with luminal compression of the IVC to $50 \%$ with partial concomitant thrombosis (Fig. 1). Moreover, the mediastinal tumor mass was now compressing the confluence of the right brachiocephalic vein and the SVC. Due to the overall constellation, thrombosis of the right internal jugular vein and partial thrombosis of the IVC, the decision was made to choose the left jugular vein access and to combine SVC stent implantation with suprarenal IVC filter insertion.

Following informed consent and local anesthesia, left internal jugular vein puncture was performed under ultrasound guidance, and a 13-Fr sheath was inserted. Bicubital mediastinal venogram confirmed compression of the confluence of the right brachiocephalic vein and of the SVC by a tumorous mass (Fig. 2). A 5000-unit bolus of heparin was

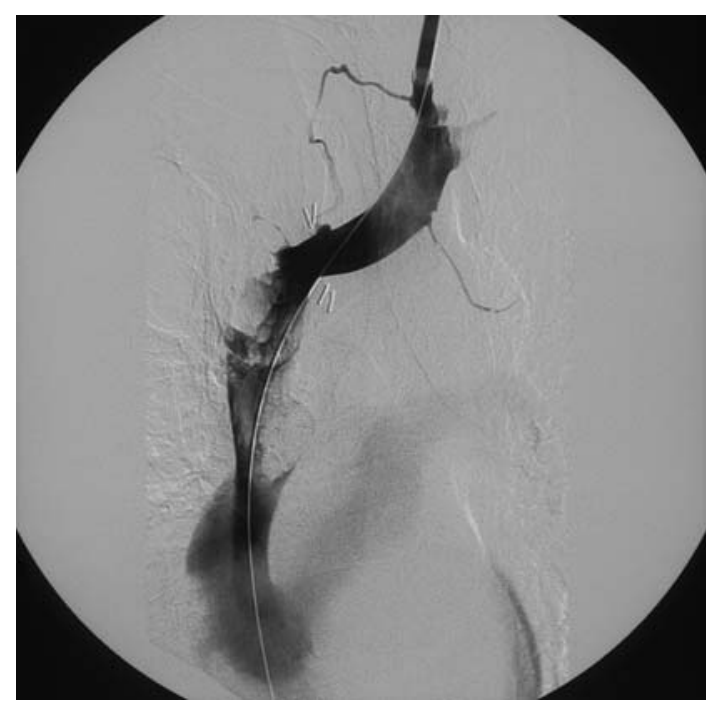

Fig. 2 Bicubital venogram demonstrates a tumorous mass lesion compressing the confluence of the right brachiocephalic vein and the SVC 


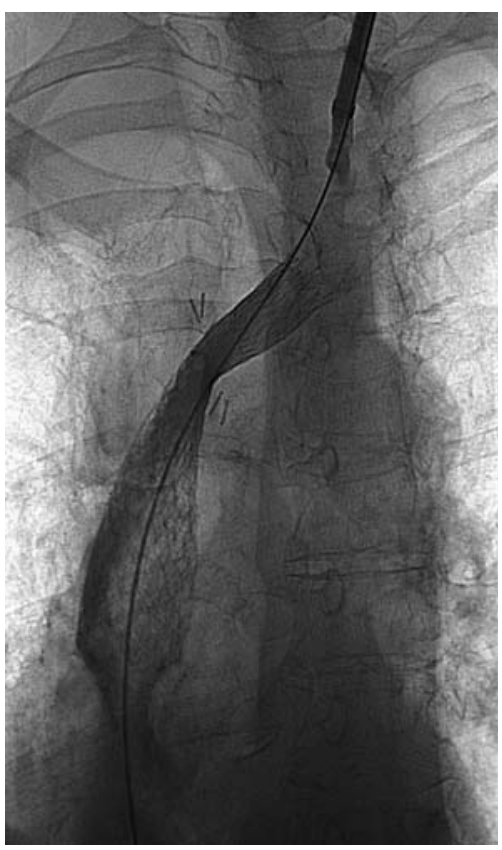

Fig. 3 Bicubital venogram after unilateral stent placement within the SVC and left brachiocephalic vein exhibits good restoration of flow

administered. After the length of the affected venous confluence had been quantified with a calibrated pigtail catheter, unilateral stent placement was undertaken. Including the left brachiocephalic vein and SVC, a selfexpanding $22 \times 90$-mm sinus-Aorta-Stent (Optimed; Ettlingen, Germany) was placed just above the right atrial level, with a good outflow of constrast medium (Fig. 3). In continuation, a femoral venogram was performed and exhibited tumor encasement of the IVC with partial thrombosis at the level of the right suprarenal vein. A retrievable vena cava filter (OPTEASE; Cordis, Roden, The Netherlands) was then successfully inserted in a suprarenal position using the left jugular access (Fig. 4). A femoral approach for inferior vena caval infiltration was avoided, so as not to trigger pulmonary embolism. At the end of the intervention, full-scale heparinization (10 units/ $\mathrm{kg}$ body weight per $24 \mathrm{~h}$ ) was initiated. Surgical port implantation was performed 1 month later, and chemotherapy was recommenced. The patient had excellent relief from her symptoms of SVCS, did not experience pulmonary embolism during a follow-up of 6 months, and is in a well general condition to date.

\section{Discussion}

Armand Trousseau was among the first authors who described the relationship between cancer and VTE [12]. Matter of factly, his theories proved to be correct, when he diagnosed himself with stomach cancer because of a

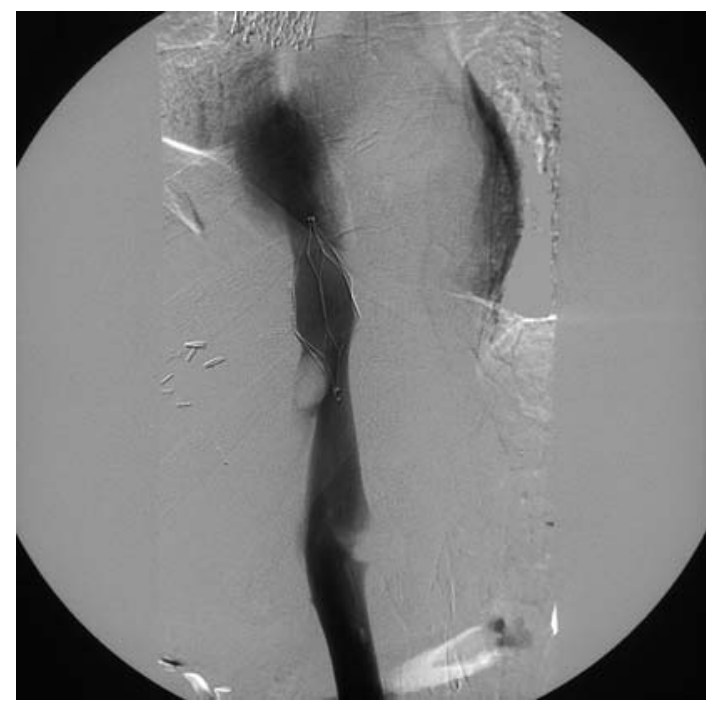

Fig. 4 Femoral venogram depicts successful suprarenal IVC filter insertion just above the thrombus formation

phlebitis on his left upper limb. In his honor, the coincidence of cancer and VTE is called Trousseau syndrome, belonging in the paraneoplastic sphere.

Jaffer et al. [2] calculated an elevated risk of thrombosis in cancer patients, underlining the topicality of this issue. Cancer patients with VTE further have an increased probability of death compared to patients without a thromboembolic event. This was proved by Sørensen et al. [4] in an age-, gender-, and diagnosis-matched study. The 1-year survival rate among cancer patients without VTE was $36 \%$, versus $12 \%$ in patients with a thrombosis.

The development of thrombi can also be potentiated by risk factors concerning treatment of the cancer [13, 14]. There are specific chemotherapeutic agents increasing the incidence of thrombotic events, for example, tamoxifen in the therapy of breast cancer or platinum-based therapies, 5fluorouracil, mitomycin, growth factors, and thalidomide. Additionally, surgery, radiotherapy, and immobility may initiate platelet aggregation [15].

Reviewing the literature for rectal, Rickles et al. [16] listed the most frequently associated tumors with venous thrombosis and revealed a $15.2 \%$ frequency for colon cancer. In addition, Levitan et al. [17] showed that even 62 of 10,000 patients with rectal cancer develop a deep venous thrombosis or pulmonary embolism. Specifically, rectal cancer patients treated with a combination of fluorouracil and leucovorin calcium, as in our case, because of colorectal have a high risk (15\%) for developing a VTE [3].

Different pathogenetic mechanisms have been described to better understand the appearance of the hypercoagulable state [18-20]. There are alterations in coagulation and anticoagulant proteins and endothelial cells due to the cytotoxic agents. In addition, the tumor itself can produce 
procoagulants and activate endogenous prothrombotic factors [5, 6].

Venous thromboembolic events include superficial and deep venous thrombosis, pulmonary embolism, and catheter-associated thrombosis [15]. While deep vein thrombosis is the most common type of thromboembolic event, SVCS is a rare complication.

A mediastinal invasion by bronchogenic carcinoma is the most common cause of SVCS, accounting for $70 \%-$ $80 \%$ of all cases [10]. In our case, secondary spread of a rectal tumor into the retrocaval, mediastinal lymph nodes caused the syndrome, with congestion, edema of the face and upper thorax, dyspnea, and dysphagia.

Different strategies for the treatment of SVCS exist [2123]. Many authors postulate chemotherapy and radiotherapy as the standard treatment for SVCS in association with malignant disease. Rodrigues et al. [21] treated SVCS with three weekly high-dose fractions of 8 Gy and achieved a partial response in $96 \%$ of all patients, and a complete response in $56 \%$. It must be considered that radiotherapy may have side effects like fever, nausea, vomiting, and mucositis, so that the patient loses further quality of life [10].

Charnsangavej et al. [22] were the first to describe SVC stenting as a further option. Since then, it has become a standard treatment for this condition, because it provides rapid relief of symptoms and the relief of SVCS in $80 \%-$ 95\% of patients [23]. However, in cases of SVCS caused by small cell carcinomas and lymphomas, chemotherapy is recommended as the first-line treatment [21,23].

Concomitant treatment with anticoagulants after SVC stenting may generally reduce the risk of deep venous thrombosis in cancer patients $[11,22,23]$; moreover, anticoagulation may induce delays of neoendothelial proliferation. However, to date, a clear consensus concerning the application and dosage of anticoagulant agents in combination with SVC stent implantation does not exist.

Parallel to stents, IVC filters have developed increasingly over the past two decades. Since their introduction in 1973, these filters have proven to be safe and effective in the prevention of life-threatening pulmonary emboli [24]. Jarrett et al. [25] have shown that even advanced-stage cancer patients may benefit from IVC filters. In a study by Wallace et al. [24] the probability of survival at 30, 90, and 365 days in patients with gastrointestinal cancer was $79 \%$, $54 \%$, and $29 \%$, respectively.

However, with regard to the clinical literature, the utility of IVC filters is not universally accepted and results are somewhat conflicting [26, 27]. In this context, a prospective, controlled clinical trial by Decousus et al. [27] reported that inferior vena caval infiltration was useful in preventing pulmonary embolism when combined with anticoagulation. Nevertheless, an improvement in overall mortality in patients with such a combined therapy did not vary significantly from these patients with anticoagulation alone. Most importantly, in patients with inferior vena caval infiltration and anticoagulation a twofold increase in recurrent deep venous thrombosis was observed in comparison to patients with anticoagulation alone.

Some authors have already reported that practice patterns concerning IVC infilration were different and that many indications for IVC filter insertion were a matter of opinion [26-28]. With respect to Trousseau's syndrome, therapy with heparin anticoagulation alone is reported to be effective and the therapy of choice [28]. Since this syndrome is a hypercoaguable state, inferior vena caval infiltration is said to be inadequate. In our case, it remains speculative if IVC thrombosis was attributable to tumor invasion or to sytemic hypercoaguability. Even though our patient had formerly experienced right jugular vein thrombosis, this complication might have been interpreted as postsurgical syndrome. Therefore, the combination of ICV filtration and anticoagulation after SVC stent implantation seemed justified for us.

In conclusion, with regard to the clinical scenario described herein, we see in SVC stenting and IVC filter implantation combined with heparin anticoagulation an efficient and safe approach to the therapy of SVCS and IVC thrombosis to pave the way for further chemotherapeutic treatment and to guarantee palliation in progressive rectal cancer. Interventional radiologists as well as clinicians should be aware of such an extraordinary course of malignant disease in order to provide effective management.

\section{References}

1. American Cancer Society (2006) Cancer facts and figures 2006. American Cancer Society, Atlanta, GA

2. Jaffer AK (2005) Issues in anticoagulant therapy: recent trials start to answer the tough questions. Cleve Clin J Med 72:157-163

3. Otten HM, Mathijssen J, ten Cate H, Soesan M, Inghels M, Richel DJ, Prins MH (2004) Symptomatic venous thromboembolism in cancer patients treated with chemotherapy: an underestimated phenomenon. Arch Intern Med 164:190-194

4. Sorensen HT, Mellemkjaer L, Olsen JH, Baron JA (2000) Prognosis of cancers associated with venous thromboembolism. N Engl J Med 343:1846-1850

5. Schafer AI, Levine MN, Konkle BA, Kearon C (2003) Thrombotic disorders: diagnosis and treatment. Hematol Am Soc Hematol Educ Program 1:520-539

6. Van Gerpen R, Mast ME (2004) Thromboembolic disorders in cancer. Clin J Oncol Nurs 8:289-299

7. Kim SH, Brennan MF, Russo P, Burt ME, Coit DG (1998) The role of surgery in the treatment of clinically isolated adrenal metastasis. Cancer 82:389-394

8. Lee JJ, Kupfer J, Raissi S, Geller SA, Siegel RJ (1998) Rapid extension of left adrenocortical carcinoma into the right atrium. J Am Soc Echocardiogr 11:86-88 
9. Zisman A, Wieder JA, Pantuck AJ, Chao DH, Dorey F, Said JW, Gitlitz BJ, deKernion JB, Figlin RA, Belldegrun AS (2003) Renal cell carcinoma with tumor thrombus extension: biology, role of nephrectomy and response to immunotherapy. J Urol 169:909-916

10. Lanciego C, Chacon JL, Julian A, Andrade J, Lopez L, Martinez B, Cruz M, Garcia-Garcia L (2001) Stenting as first option for endovascular treatment of malignant superior vena cava syndrome. AJR 177:585-593

11. Marcy PY, Magne N, Bentolila F, Drouillard J, Bruneton JN, Descamps B (2001) Superior vena cava obstruction: Is stenting necessary? Support Care Cancer 9:103-107

12. Trousseau A (1865) Phlegmasia alba dolens. In: Trousseau A (ed) Clinique medicale de l'Hotel-Dieu de Paris, Vol. 3. Balliere, Paris, pp 654-712

13. Saphner T, Tormey DC, Gray R (1991) Venous and arterial thrombosis in patients who received adjuvant therapy for breast cancer. J Clin Oncol 9:286-294

14. Viale PH, Schwartz RN (2004) Venous thromboembolism in patients with cancer. Part II. Current treatment strategies. Clin J Oncol Nurs 8:465-469

15. Cunningham RS (2005) Therapeutic options for the treatment of cancer-associated thrombosis. Semin Oncol Nurs 21(Suppl 1): $21-40$

16. Rickles FR, Edwards RL (1983) Activation of blood coagulation in cancer: Trousseau's syndrome revisited. Blood 62:14-31

17. Levitan N., Dowlati A, Remick SC, Tahsildar HI, Sivinski LD, Beyth R, Rimm AA (1999) Rates of initial and recurrent thromboembolic disease among patients with malignancy versus those without malignancy. Risk analysis using Medicare claims data. Medicine (Baltimore) 78:285-291

18. Kuzel T, Esparaz B, Green D, Kies M (1990) Thrombogenicity of intravenous 5-fluorouracil alone or in combination with cisplatin. Cancer 65:885-889
19. Feffer SE, Carmosino LS, Fox RL (1989) Acquired protein C deficiency in patients with breast cancer receiving cyclophosphamide, methotrexate, and 5-fluorouracil. Cancer 63:1303-1307

20. Bertomeu MC, Gallo S, Lauri D, Levine MN, Orr FW, Buchanan MR (1990) Chemotherapy enhances endothelial cell reactivity to platelets. Clin Exp Metastasis 8:511-518

21. Rodrigues CI, Njo KH, Karim AB (1993) Hypofractionated radiation therapy in the treatment of superior vena cava syndrome. Lung Cancer 10:221-228

22. Charnsangavej C, Carrasco $\mathrm{CH}$, Wallace $\mathrm{S}$, Wright $\mathrm{KC}$, Ogawa K, Richli W, Gianturco C (1986) Stenosis of the vena cava: preliminary assessment of treatment with expandable metallic stents. Radiology 161:295-298

23. Uberoi R (2006) Quality assurance guidelines for superior vena cava stenting in malignant disease. Cardiovasc Interv Radiol 29:319-322

24. Wallace MJ, Jean JL, Gupta S, Eapen GA, Johnson MM, Ahrar K, Madoff DC, Morello FA, Murthy R, Hicks ME (2004) Use of inferior vena caval filters and survival in patients with malignancy. Cancer 101:1902-1907

25. Jarrett BP, Dougherty MJ, Calligaro KD (2002) Inferior vena cava filters in malignant disease. J Vasc Surg 36:704-707

26. Henkle G, Kunz P, Funaki B (2004) Patterns of referral for inferior vena caval filtration: delays and their impact. AJR 183:1021-1024

27. Decousus H, Leizorovicz A, Parent F, et al. (1998) A clinical trial of vena caval filters in the prevention of pulmonary embolism in patients with deep venous thrombosis prevention du risque d'embolie pulmonaire par interruption. Cave Study Group. N Engl J Med 338:409-415

28. Hann CL, Streiff MB (2005) The role of vena caval filters in the management of venous thromboembolism. Blood Rev 19:179202 